Trauma Berufskrankh $2008 \cdot 10$

[Suppl 1]:112-116

DOI 10.1007/s10039-007-1260-1

Online publiziert: 15. Dezember 2007

(c) Springer Medizin Verlag 2007
T. Schmickal ${ }^{1} \cdot A$. Wentzensen ${ }^{2} \cdot \mathrm{K}$. Weise ${ }^{3}$

${ }^{1}$ Unfallchirurgische Klinik, Klinikum Neumarkt, Neumarkt i.d.Opf.

${ }^{2}$ BG-Unfallklinik, Ludwigshafen

${ }^{3}$ BG-Unfallklinik, Tübingen

\section{Kompetenzzentrum zur Rehabilitationsabklärung}

\author{
Ein Konzept des Landesverbands \\ Südwestdeutschland der gewerblichen Berufs- \\ genossenschaften zur frühzeitigen Evaluation \\ und Beurteilung der Erfolgsaussichten der \\ beruflichen Rehabilitation Unfallverletzter in den \\ BG-Unfallkliniken Ludwigshafen und Tübingen
}

\section{Hintergrund und Fragestellung}

Derzeit wird das berufsgenossenschaftliche Heilverfahren in der Regel von den durchführenden Kliniken und ambulant tätigen D-Ärzten geführt. Überwacht und gesteuert wird es von den gesetzlichen Unfallversicherungsträgern unter Konsultation der Beratungsärzte. Erfahrungen in BGSW-Kliniken zeigen, dass bei einem bestimmten Prozentsatz der Versicherten alle Rehabilitationsbemühungen zum Scheitern verurteilt sind, da einerseits aufgrund des Verletzungsmusters die Rehabilitationsfähigkeit nicht oder noch nicht gegeben ist, andererseits aber auch bei manchen Versicherten die innere Bereitschaft zur Wiederaufnahme der Arbeitstätigkeit fehlt. Diese Einschätzungen können weder durch den Beratungsarzt noch durch Heilverfahrenskontrollen in entsprechenden Zentren (BG-Kliniken) bei einer einmaligen und zeitlich limitierten Vorstellung getroffen werden.

Das neue vorliegende Konzept bietet den Versicherungsträgern an, dass ein im berufsgenossenschaftlichen Heilverfahren erfahrenes Team, bestehend aus Arzt, Physiotherapeut und Psychologe, ggf. ergänzt um Schmerztherapeut bzw. Berufshilfe- und Sozialdienst, im Rahmen ei- ner 3-tägigen Untersuchung mit psychologischer Evaluation des Patienten eine frühzeitige Stellungnahme zur Prognose des Heilverfahrens abgibt und einen konkreten Rehabilitationsplan erstellt, ggf. ergänzt durch die Einbindung von Konsiliarärzten wie Arbeitsmedizinern und anderen Fachspezialisten. Bei Bedarf werden zusätzlich arbeitsplatzbezogene Assessmentverfahren (ERGOS ${ }^{\oplus}, \mathrm{EFL}^{\circledast}$ ) eingeleitet. Es werden auch Angaben darüber gemacht, ob das Heilverfahren Chancen auf eine erfolgreiche berufliche Reintegration bietet.

Gerade in Zeiten des zunehmenden Kostendrucks im Gesundheitssystem soll dieses Projekt den Kostenträgern ein Instrument zur Verfügung stellen, das helfen soll, ineffiziente Behandlungsmaßnahmen im Heilverlauf zu minimieren und nicht Erfolg versprechende Reintegrationsmaßnahmen zu vermeiden. Das Projekt soll es den Kostenträgern ermöglichen, frei werdende Ressourcen zum Wohl der hinsichtlich der Rehabilitation hochmotivierten Unfallverletzten zur Steigerung der Effizienz des Heilverfahrens einzusetzen.

\section{Methode des neuen Konzepts}

Die Rehabilitationsabklärung erfolgt durch:

1. Umfassende Befundaufnahme der Verletzungsfolgen durch ein Evaluationsteam bestehend aus

- Arzt

- Psychologe

- Physiotherapeut

- ggf. Schmerztherapeut

- ggf. Berufshilfe-/Sozialdienst

2. Analyse des bisherigen Status und Prognose der zu erwartenden Verletzungsfolgen

3. Psychologische Evaluation der Einstellung und Erwartungshaltung des Verletzten, der psychischen Komorbiditäten und seiner Motivation im Hinblick auf den Genesungsverlauf und die berufliche Wiedereingliederung

4. Physiotherapeutische Befundaustestung mit Teilnahme an Einzeltherapie, Gruppentherapie, isokinetischer Untersuchung, Worksimulator

5. Bei Bedarf Einschaltung von Konsiliarärzten/Arbeitsmediziner gemäß Konzept des LVBG

6. 3-tägiger stationärer Aufenthalt in der BG-Klinik Ludwigshafen oder Tübin- 
gen mit entsprechender Unterbringung und Verpflegung

7. Umfassender Befundbericht an den Kostenträger durch das Evaluationsteam

- Empfehlung medizinischer Maßnahmen

- Ausführlicher Rehabilitationsplan

- Prognose der Reintegrationsfähigkeit und eventueller psychischer Barrieren (Verletzungsmuster, Motivation)

- Empfehlung für den Berufshelfer (ABE-Plan, berufliche Empfehlung)

Die Kliniken bieten außerdem eine offen ausgesprochene und von mehreren Fachgruppen erarbeitete Empfehlung, ob und welche weiteren Maßnahmen durch die Kostenträger auf ein notwendiges Minimum begrenzt werden sollen, wenn Verletzungsmuster, Befund, Motivation und privates sowie berufliches Umfeld eine Reintegration des Verletzten nicht mehr erwarten lassen!

\section{Indikation zur Vorstellung}

Eine möglichst frühzeitige Rehabilitationsabklärung kann insbesondere in folgenden Fällen angezeigt sein:

- Überschreitung der normalen Dauer der Arbeitsunfähigkeit, wenn z. B. der Wiedereintritt der Arbeitsfähigkeit nicht absehbar ist

- Kein erkennbarer Zusammenhang zwischen geklagten Beschwerden und Arbeitsunfähigkeit

- Abstimmung von arbeits- bzw. arbeitsplatzbezogenen Maßnahmen

- Probleme bei der Arbeits- und Belastungserprobung

- Komplikationsdiagnosen im Zusammenhang mit der Unfallverletzung

- Komplikationsdiagnosen unabhängig von der Unfallverletzung (Suchtproblematik usw.)

- Klärung weiterer Behandlungsmaßnahmen (EAP, BGSW, Schmerztherapie)

- Vorstellung vor größeren Eingriffen (z. B. TEP im Bereich Hüfte oder Knie)

- Festlegung der erforderlichen orthopädietechnischen Versorgung

Trauma Berufskrankh 2008 · 10[Suppl 1]:112-116 DOI 10.1007/s10039-007-1260-1

(c) Springer Medizin Verlag 2007

T. Schmickal · A. Wentzensen $\cdot$ K. Weise

Kompetenzzentrum zur Rehabilitationsabklärung. Ein Konzept des Landesverbands Südwestdeutschland der gewerblichen Berufsgenossenschaften zur frühzeitigen Evaluation und Beurteilung der Erfolgsaussichten der beruflichen Rehabilitation Unfallverletzter in den BG-Unfallkliniken Ludwigshafen und Tübingen

\section{Zusammenfassung}

Die kurzstationäre Evaluation in den Kompetenzzentren Rehabilitationsabklärung der BG-Unfallkliniken Tübingen und Ludwigshafen verfolgt das Ziel, bei Arbeitsunfallverletzten mit verzögertem oder unklarem Heilverlauf und/oder Problemen der beruflichen Wiedereingliederung eine ausführliche medizinische bzw. medizinisch-berufsbezogene und diagnostische Abklärung durchzuführen. Durch ein interdisziplinäres Evaluationsteam soll unter Berücksichtigung des aktuellen Leistungsvermögens, unabhängig vom Zeitpunkt des Behandlungsgeschehens und der Durchführung anderer ambulanter und/ oder stationärer Maßnahmen, wie EAP, BGSW und Wiederholungsheilverfahren, eine umfassende Abklärung der Rehabilitationsmöglichkeiten erfolgen. Als Vorschlag für den Unfallversicherungsträger wird ein individuelles, zielgerichtetes und mit Zeitvorgaben versehenes Rehabilitationskonzept mit Empfehlungen zu weiterführenden medizinischen und/oder berufsbezogenen Maßnahmen erstellt. Das Verfahren soll damit eine effizientere Steuerung im berufsgenossenschaftlichen Heilverfahren durch Reduktion ineffizienter Behandlungsmaßnahmen, aber auch eine Umverteilung vorhandener Ressourcen zur effizienten Bündelung der Behandlungsmaßnahmen und raschen beruflichen Reintegration des Verletzten ermöglichen.

\section{Schlüsselwörter}

Kurzstationäre Evaluation · Interdisziplinäres Evaluationsteam · Rehabilitationsmöglichkeiten · Rehabilitationskonzept . Heilverfahrenseffizienz

\section{Centre of excellence for evaluation of rehabilitation. A concept of the Southwest Germany division of the employers' liability insurance association for early evaluation and assessment of the prospects of success in occupational rehabilitation of accident victims in the association's trauma hospitals in Ludwigshafen and Tübingen}

\section{Abstract}

The aim of the brief in-patient evaluation in the centres of excellence for rehabilitation questions in the employers'liability insurance association's trauma hospitals in Ludwigshafen and Tübingen is a detailed medical, or rather medical/occupational, and diagnostic investigation in patients who have had occupational accidents followed by a delayed or non-straightforward course of recovery and/or problems with reintegration into working life. The interdisciplinary evaluation team is expected to carry out a comprehensive investigation into the options for rehabilitation, with due consideration for the patient's current capacity, regardless of the timing of the treatment and the implementation of other out-patient or in-patient procedures, such as extended ambulant physiotherapy, further in-patient treatment and repeat admission for new treatment following objective worsening of any disablement. An individual targeted rehabilitation plan is prepared, with times for each step and with recommendations for further treatments and occupation-related elements, and is suggested to the accident insurance company. This process is intended to enable more efficient control of treatments approved by the employers'liability insurance association, partly by reducing the number of ineffective treatments but also by redistributing the available resources to concentrate treatments efficiently and achieve rapid reintegration of accident victims into working life.

\section{Keywords}

Brief in-patient evaluation - Interdisciplinary evaluation team - Rehabilitation options . Rehabilitation plan - Efficacy of treatment 
- Schwere neurologisch-traumatologische Fälle

- Fälle mit Problematiken nach $\$ 46$, Abs. 3 SGB VII (78. Woche), Beurteilung der Arbeitsunfähigkeit/Erwerbsunfähigkeit

\section{Benötigte Unterlagen}

Für eine Evaluation des Versicherten in der vorbeschriebenen Weise werden vom UV-Träger benötigt:

- komplette Krankenunterlagen

- komplettes Vorerkrankungsregister

- Stellungnahme des Berufshelfers bezüglich Arbeitsplatz und Reintegrationsmöglichkeiten durch Fragebogen

\section{Zeitplan der Standardevaluation}

\section{Tag}

\section{Vormittag}

- Stationäre Aufnahme

- Eingangsuntersuchung durch den Stationsarzt und den Physiotherapeuten

- Einteilung und Beginn der psychosozialen Evaluation durch den Psychologen

- Aushändigung der psychometrischen Diagnoseverfahren an den Versicherten

- Erstellung des Untersuchungsplans und Gruppeneinteilungen (Einteilung erfolgt durch den Physiotherapeuten)

\section{Nachmittag}

- Teilnahme an der physiotherapeutischen Gruppentherapie

- ggf. isokinetische Untersuchung (Cybex)

- bei Bedarf hausinterne schmerztherapeutische Konsilanforderung

\section{Tag}

\section{Vormittag}

- Fortsetzung der psychosozialen Evaluation durch den Psychologen

- Auswertung der psychometrischen Testung

- Stellungnahme

\section{Nachmittag}

- Erhebung der Sozialanamnese durch Psychologe/Berufshilfe-/Sozialdienst

- Teilnahme an der physiotherapeutischen Gruppentherapie

- ggf. ergänzende Testung am Worksimulator

\section{Tag}

\section{Vormittag}

- Teilnahme an der Gruppentherapie

- ggf. isokinetische Zusatzuntersuchung

\section{Mittag}

- gemeinsame Besprechung von Stationsarzt, Psychologe, Physiotherapeut, ggf. Berufshilfe-/und Sozialdienst und Ausarbeitung einer Stellungnahme mit Prognose, ggf. mit ausführlichem Rehabilitationsplan

- bei positiver Prognose, sofern vom Kostenträger erwünscht, Terminvereinbarung zur Übernahme in das BGSW-Verfahren

\section{Abschluss}

- Besprechung und Übergabe eines Rehabilitationsplans an den Versicherten zur Weiterleitung an die weiterbehandelnde Institution

- Sofortinformation an den Kostenträger (z. B. Fax)

- Abreise

\section{Inhalte der Untersuchungseinheiten}

\section{Medizinischer Merkmalsbereich}

Der ärztliche Aufnahmebefund erstellt ein umfassendes Profil der stattgehabten Verletzungen, ihrer Versorgung und aktuell bestehender Folgen und stellt damit die Basis zur Einschätzung der weiteren Prognose des Heilverlaufs dar. Dies erfolgt durch:

- Unterlagenbewertung der Vorbehandlung

- klinische Befundaufnahme

- Bewertung vorliegender Untersuchungsbefunde und bildgebender Verfahren

- Einordnung in ein Zeitraster („Stand des Heilverfahrens") unter Hinzuzie- hung bekannter Parameter, wie „Weller-Tabelle“

\section{Physio- bzw. ergotherapeutischer Merkmalsbereich}

Die physiotherapeutische Untersuchung verfolgt das Ziel, ein aussagekräftiges Bild über die infolge der Verletzung verbliebenen Defizite und der Prognose des zu erzielenden Ergebnisses mit und ohne weitere Behandlungsmaßnahmen auf physiotherapeutischem Sektor zu erstellen, wobei Befunde aus der Teilnahme an Gruppentherapien und geräteunterstützte Untersuchungen (wie die Cybex-Kraftmessung oder die Podografie) in die physiotherapeutische Einschätzung mit einbezogen werden.

Die Befunderhebung basiert auf den theoretischen Grundlagen der Anatomie, Physiologie, Neurophysiologie, Biomechanik und der Trainingslehre. Im Fokus des Evaluationsverfahrens stehen das Bewegungssystem, die körperliche Leistungsfähigkeit und die Bewegungskontrolle.

\section{Evaluationsverfahren}

\section{Bewegungssystem.}

- Gelenkbeweglichkeit nach der Neutral-o-Methode (goniometrisch) „Jrom-Test“

- Dehnfähigkeit (muskulär, ligamentär, kapsulär) - Nomenklatur der Manuellen Therapie

- Muskelkraft (manuelle Muskeltests nach Daniels u. Worthingham [1], Kendall et al. [2])

\section{Leistungsfähigkeit. \\ - Kondition \\ - Kraftausdauer}

Bewegungskontrolle.

- Koordination (intra- und intermuskulär)

\section{Gruppentherapie.}

- Gehschule

- Medizinische Trainingstherapie (Cybex-Kraftmessung)

- Fußgruppe/Fußbelastungsgruppe

- Beingruppe/Beinbelastungsgruppe

- Armgruppe/Armbelastungsgruppe

- Konditions- und Ausdauertraining 


\section{Psychologischer Merkmalsbereich}

Die psychologische Evaluation besteht in einer umfassenden Testserie, die sowohl zeit- als auch situationsübergreifende Persönlichkeits- und Krankheitsvariablen, hier besonders die Schmerzverarbeitung, erfasst. Auch die zu antizipierenden situativen Belastungen werden nicht außer Acht gelassen, um den komplexen Anforderungen, die an den Verletzten herangetragen werden, gerecht zu werden.

Patientinnen und Patienten unterscheiden sich stark in der Akzeptanz, Durchführung und Fortsetzung therapeutischer und rehabilitativer Maßnahmen. Das gilt auch für die Bewältigung der Beschwerden, die Motivation zum Durchhalten, den Genesungsverlauf, die Selbstwirksamkeit bei der Krankheitsbewältigung, die Bewältigungsstrategien bei Belastungen und die Compliance (Motivation zur Zusammenarbeit). So können unterschiedliche Selbsteinschätzungen, Motivation und Aktivitäten der Patienten zur Erreichung der therapeutischen und rehabilitativen Ziele dazu führen, dass spezifische Maßnahmen und Stützungshilfen im Verlauf des Heilverfahrens (und evtl. danach) entwickelt und eingesetzt werden, damit eine größtmögliche Effektivität und Nachhaltigkeit des Rehabilitationsprogramms erzielt werden können.

Darüber hinaus sind Effekte auf den Heilungsverlauf durch (relativ) zeitinvariante Persönlichkeitsmerkmale zu beachten.

Psychologische Merkmale spielen in der Schmerzbewältigung eine wesentliche Rolle. Als gesichertes Forschungsresultat gilt beispielsweise, dass Depressivitätstendenzen beim Umgang mit den Schmerzen und bei der Wiedereingliederung in das Berufsleben von erheblicher (negativer) Effektivität sein können.

Neben überdauernden psychologischen Merkmalen (Eigenschaften) sind im Kontext der Rehabilitation von Unfallnoxen die Bewältigungsformen von spezifischen, mit den Unfallschädigungen zusammenhängenden, bereits erlebten oder noch zu antizipierenden Belastungen relevant. So lässt sich vermuten, dass Patienten mit ausgeprägt pessimistischen Antizipationstendenzen sicher an das Eintreten belastender, demütigender und auslie- fernder Situationen glauben und diese sogar möglicherweise suchen oder herbeirufen mit der möglichen Folge einer Negativübertragung auf die Bemühungen zur beruflichen Wiedereingliederung (Motto: "Zwecklos!“).

Folgende relevante und wissenschaftlich begründete psychologische Merkmalsbereiche sollten singulär und kombiniert im Hinblick auf die Stärke ihrer Einflussnahme auf den Heilungsprozess analysiert werden:

- psychosomatische und psychische Störungen, Persönlichkeitsfaktoren

- Umgang mit dem Unfall, den Verletzungen, den Handicaps ( $\rightarrow$ Lebensqualität)

- Körperbild/Körperschema (Störung)

- Bewältigungsstrategien und Motivation zur Zusammenarbeit

- soziale Unterstützung

- Selbstwirksamkeit bei der rehabilitativen Zielerreichung

- eigene Lebensperspektive/Konkretheit von Lebensperspektiven

— Depressivität, Ängste

- Motivation (im Stadienverlauf), die Rehabilitationsmaßnahmen kooperativ zu unterstützen

- (aktueller) Substanzmissbrauch

- aktuelle Art und Stärke von Schmerzen

- subjektive Retrospektion über Erfolg oder Misserfolg bisheriger Rehabilitationsmaßnahmen

- Gründe für evtl. Unterbrechung der AF (objektiv und seitens des Patienten)

- Gründe für evtl. misslungene ABE (objektiv und seitens des Patienten)

- vergangene oder laufende juristische Verfahren

- besondere kritische Lebensereignisse

\section{Sozialer Merkmalsbereich}

Die soziale Anamnese komplettiert die Evaluation. Sie erhebt die allgemeinen soziodemografischen Daten, erfasst durch Patientenunterlagen oder anamnestische Exploration wie

- Lebensalter

- Geschlecht

- Bildungsniveau (Schule)

- Berufsausbildung

- familiärer Status (ggf. Kinderzahl)
- ständige Personenzahl des Haushalts - gegenwärtiger Status des Arbeitsverhältnisses

Zudem werden Aspekte der Arbeitsfähigkeit, der durchgeführten Arbeits- und Belastungserprobungen und der sozialen Unterstützung berücksichtigt:

- allgemein belastende Lebensbedingungen

- Zufriedenheit im Beruf

- familiäre Verpflichtungen

- Schwerbehindertenstatus/Gleichstellung

- Rentenverfahren

- Dauer der Arbeitsunfähigkeit

- bisherige berufshelferische Interventionen

- vorhandene (oder zu erwartende) soziale Unterstützung

Die Daten der sozialen Merkmalsbereiche werden mit denen der anderen Evaluationsebenen in Bezug gesetzt.

\section{Abklärung der Indikation von Schmerztherapie}

Anhaltende Schmerzen werden von vielen Patienten nach einem Unfall beschrieben. Dieses oft komplexe Beschwerdebild erfordert eine interdisziplinäre Erfassung durch die an der Rehabilitationsabklärung beteiligten Fachspezialisten. Neben Schmerzort(en), Intensität und Auftretenshäufigkeit werden schmerzbedingte Einschränkungen von Mobilität und Befinden erfasst. Ergibt die Evaluation die Notwendigkeit einer weitergehenden Schmerzdiagnostik, wird ein schmerztherapeutisches Konsil im Hause veranlasst zur Klärung der Frage, in welchem Rahmen Schmerztherapie in die Rehabilitationsplanung integriert werden sollte.

\section{Arbeitsplatzspezifische Diagnostik}

Sie wird im Rahmen der stationären Rehabilitationsbehandlung in den BG-Unfallkliniken bereits seit langer Zeit eingesetzt. Zur Verfügung stehen eine große Anzahl unterschiedlicher Arbeitsplätze in Metall- und Holzwerkstätten, Worksimulatoren sowie Geländesimulationsstrecken, die eine individuelle Testung 
verbliebener Defizite unter Berücksichtigung der arbeitsplatzspezifischen Situation erlauben.

\section{Fazit für die Praxis}

Das Verfahren soll unter Nutzung verschiedener Untersuchungsmethoden eines interdisziplinären Teams aktuelle und valide Aussagen zum Stand verzögerter oder drohend zu entgleisender Heilverläufe liefern und damit durch Reduktion ineffizienter Behandlungsmaßnahmen, aber auch eine Umverteilung vorhandener Ressourcen zur effizienten Bündelung der Behandlungsmaßnahmen und raschen beruflichen Reintegration des Verletzten eine effizientere Steuerung im berufsgenossenschaftlichen Heilverfahren ermöglichen.

\section{Korrespondenzadresse}

Dr. T. Schmickal

Unfallchirurgische Klinik, Klinikum Neumarkt, 92381 Neumarkt i.d.Opf.

Thomas.schmickal@klinikum-neumarkt.de

Interessenkonflikt. Keine Angaben

\section{Literatur}

1. Daniels L, Worthingham C (1992) Muskeltest Manuelle Untersuchungstechniken. Fischer, Stuttgart

2. Kendall FP,McCreary-Kendall E, Provance PG (1993) Muscles testing and function: with posture and pain. Williams \& Wilkins,Baltimore 